\title{
New Trends in Mobility Modelling and Handover Prediction
}

\author{
Enrica Zola ${ }^{1}$, Francisco Barcelo-Arroyo ${ }^{1}$, Israel Martin-Escalona ${ }^{1}$, Andrea G. \\ Ribeiro $^{2}$, Rute Sofia ${ }^{2}$, Krzysztof Grochla $^{3}$, Michal Gorawski ${ }^{3}$, Konrad Polys ${ }^{3}$ \\ 1 Universitat Politècnica de Catalunya (UPC), Spain \\ ${ }_{2}^{2}$ COPELABS, University Lusofona, Portugal \\ ${ }^{3}$ Institute of Theoretical and Applied Informatics of PAS, Gliwice, Poland
}

\begin{abstract}
A wireless network may include fixed nodes and mobile nodes that change the location during data transmission. Node mobility has significant impact on the operation of a wireless network, as the signal propagation conditions depend on the location of the nodes and may cause dramatic changes in the data transmission rates and packet error rates. Because the network performance is influenced by the location and signal propagation conditions between network nodes, accurate representation of the user mobility in the wireless network analysis is a crucial element in both simulation and numerical or analytical modelling. This chapter discusses mobility models used today to simulating mobile network behavior. Further, the handover optimization and prediction are discussed, along with alternative methods of radio signal propagation changes caused by personal mobility.
\end{abstract}

Keywords: mobility modeling; prediction.

\section{Introduction}

Wireless technology is today omnipresent as complementary access in current telecommunication technology, being used in a wide range of network architectures, ranging from infrastructure-mode hotspots to pervasive mesh networks. The deployment of wireless community networks relies on short- and long-range wireless technologies. Reasons for integrating wireless include the low-cost of short-range wireless technology; the evolution of wireless end-user devices, as well as the emergence of smart Access Points (APs) that allow for spontaneous growth of community networks. Reasons for this heavy deployment relates to the low-cost of short-range wireless and also to the evolution of wireless enduser devices, as well as to the emergence of smart APs, that allow community networks to grow spontaneously.

From the operational perspective, these network architectures should not oversee an important component: wireless devices are carried by humans, thereby inheriting human mobility patterns which impact network operation and performance. First and foremost, mobility heavily impacts path re-computation as 
multihop routing is based on the single-source shortest path paradigm. Secondly, resource management is affected, particularly in dense networks which naturally arise in highly populated areas such as cities. Here, it is not uncommon to have an AP per household, which serves a few users and a few devices.

Besides the path re-computation, predictability of the whereabouts of Mobile Nodes ( $M N s$ ) can help in adaptation of the mobile service, which should be flexible enough to work with different transmission links, all the more so because a single user can have a device with more than one wireless interface.

In a mobile network environment, the MNs' movement path has impact on several aspects of networking, which range from location update and paging, radio resource management (e.g. dynamic channel allocation schemes), to technical network planning and design (e.g. cell and location area layout, network dimensioning).

This chapter discusses trends in mobility modelling as a tool that is currently being applied in simulations concerning experimentation of mobile network behavior, as well as in handover optimization and prediction along with alternative methods of radio signal propagation changes caused by personal mobility.

The chapter is organized as follows. Section 2 introduces relevant aspects to the context of mobility modeling, while section 3 describes related work focused on obtaining good quality movement traces that can be used in modelling mobility in cellular networks. Section 4 is dedicated to prediction aspects applied to cellular networks. In section 5 we also propose methods to simplify evaluation of the network and comparison of real GPS traces with synthetic mobility traces, by directly modulating the transmission bitrate and packet loss rate in the simulation without full representation of client location. Section 6 summarizes the chapter.

\section{Background on Mobility Modeling}

A better understanding of the node movement and, if possible, the transfer of specific and common properties to the several aspects of network operation facilitate optimization of the network performance. Traces, investigated and gathered by the scientific community, assist in understanding the mobility behaviour of nodes under different conditions and, from the analytical perspective, may be useful when modelling such movement behaviour. From the perspective of wireless and cellular network operation, it is essential to quantify the human movement behaviour, given that the paradigms described are based on Internet end-users carrying and/or owning a multitude of wireless enabled devices. These devices have a movement pattern similar to their human carriers, therefore the movement of these devices is affected by the human interest to socialize and cooperate.

In addition to obtaining the node behaviour from real traces, for many years there has been an attempt to define the movement of nodes synthetically. In networking, most of such attempts are based on the Bayesian [21] models mostly 
because of its capability to provide some variability to the environments being tested.

Accurate representation of the user mobility in wireless networks analysis is a crucial element in both simulation and numerical or analytical modelling. Mobility models allow generation of the changing location of the network nodes in time. In most cases, the network area is represented by a two dimensional plane [5], although some of the models (e.g. [30]) represent the user mobility in three dimensions. The mobility model provides the coordinates of the modelled nodes in space and their evolution in time.

Simulations of mobile and wireless networks allow testing of the developed protocols in various conditions, represented in the computer memory. The simulation must have representation of the location in space for every network node to calculate the radio signal propagation. Mobility models are used for this purpose. In discrete event simulation, the model generates location of every device in the network for the time of each event in the simulation.

wireless networks analysis and experimentation, mobility models are therefore being applied to assist in experimentation concerning location updates, load balancing, radio resource management and network planning. Mobility modelling is also crucial for calculation of the probability of rare events and evaluation of wireless networking protocols' efficiency.

In what concerns mobility patterns, realistic development requires a statistical integration of realistic properties of the roaming behavior of MNs, which today is being achieved via trace-based models as well as via the development of synthetic models.

Traces can be derived from observation of real movements. To achieve this, the user log traces should be collected during a period which is long enough to capture periodical behaviours. However, exhaustive traces are hard to capture and as consequence, most models derived from traces are only applicable in specific contexts. While synthetic models attempt to statistically capture movement behavior of MNs. Speed; direction; pause time; inter-contact times are often parameters used in modeling movement. Of course, the mobility pattern derived from this analysis is quite simplistic and hence, today are models - social mobility models - that attempt to integrate social aspects into models, such as social attractiveness, as discussed in section 3.2.

The first goal is to achieve the statistical properties of the movement as similar to the ones observed in the real world as possible. The second goal is to keep the model relatively simple and easy to implement. Selection of the model has significant influence on the results. When the model is too simple or represent some properties which are not observed in the real network, its results can lead to false conclusions. The mobility models are also used while selecting parameters for the networking devices. In e.g. LTE networks, the Handover (HO) thresholds and neighbour relations are configured by the network administrator. They may be selected based on the observed traffic and network statistics or by offline analysis, which requires the modelling of the people movement. 
Another application of mobility models relates with their capability to assist in estimating specific aspects of future roaming behavior. For instance, in dynamic wireless environments of today are based on the notion that users carry low-cost and limited capacity portable devices which are cooperative in nature and which extend the network in a user-centric way, not necessarily implying the support for networking services such as multi-hop routing [12]. For instance, in user-centric networking, transmission can simply be relayed as based on simple mechanisms that already exist in end-user devices. In terms of mobility and addition to the currently available solutions, the user-centric networking is focused on two main aspects: mobility tracking and estimation, and HO support. One of the aspects described through the subsequent sections of this book is the ways of addressing patterns of node movement to estimate mobility patterns based on the existing or novel social models.

A final thing to consider is ensuring that the functionality to be developed can assist in dealing with the unmanaged aspects of dynamic wireless architectures, which requires more support for the management of mobility anchor.

HOs across user-centric networks require more support than the ones available in today's wireless technology. For instance, the regular 250 meter range of $\mathrm{Wi}-\mathrm{Fi}$ is small compared to the geographic distances that users are expected to travel in user-centric scenarios. Hence, performing a complete HO would impose strong requirements on the speed of message exchange. One way to tackle this issue is to consider the regularity (routine) of the users' movement, which may facilitate determining the place and type of resources that may be required for seamless HOs. For example, based on movement analysis, the system may determine with a high probability that the user will hand over towards the range of specific mobility anchor points. In this case, specific functions may assist in defining the next adequate target and the way of handing over to it. The challenge here is to identify with enough accuracy and reliability the gateways which a wireless node should connect to while moving.

\section{Review and Comparison of Available Mobility Models}

In this section we try to enumerate most widely used and representative models which have been categorized into different groups. For instance, Bai et al. consider mobility models to be divided into 4 categories [3] : random models; models with temporal dependencies; models with spatial dependencies; models with geographic restrictions. While pure random models are incapable of simulating complex human movement faithfully, models with certain types of dependencies provide some representation of rational human behaviours, like grouping, inertia or avoiding obstacles; but they do not model social interactions, which are described in the section 3 of this chapter. Thus we divide the models into the ones based on randomness (random models); the ones based on social interaction (social mobility models) and the models which merge these two approaches (hybrid models), respectively describing each category in sections $2.1,2.2$ and 2.3 respectively. 


\subsection{Random Models}

Random models consider Brownian processes. The periods of movement may be interrupted by periods of inactivity. The most widely used models are the basic ones. They focus mostly on randomness of human walk, like Markovian mobility, Random Waypoint (RWP) model or Random Walk (RW) [3], [42] model, and clearly are not precise enough. There are several studies [38], [39] which prove that human walk resembles Levy flight, which has been identified initially in animal movement patterns (e.g. albatrosses, insects, jellyfish, sharks) [24], [46]. Individual walking is hardly random incorporating instead some characteristics of a Levy flight [38]. Moreover, Mascolo et al. addressed social notions such as social attractiveness [34] to bring mobility modeling closer to human movement and incorporated features concerning social interaction to provide clustering of nodes in movement. Lee et al. propose several enhancements of the Levy flight pattern to give a more realistic representation of human walk [32], [38], [39] including pause-time, LATP (Least Action Trip Planning), hotspots attraction and social dependencies. Further research [14] shows that it is quite possible to obtain a mobility model with a high predictability rate, as human movement is actually less random and more regular than we think.

\section{Random Walk Model}

The simplest random mobility model is the Random Walk Model (RWM) [3], [42] which was commonly used to mimic random behaviour of particles in physics. It is characterized by pure randomness of both the movement speed and direction. The speed is selected from the $\left[V_{\min }, V_{\max }\right]$ set and the direction is selected from $[0,2 \pi]$ using uniform distribution. Typically, MNs move in one direction for a determined distance or in a fixed time interval and during each step, the speed and direction are chosen randomly. The RW is a memoryless process, where the next action is irrelevant to the previous speed and direction. No information about the previous movement is stored. It leads to obtaining totally unrealistic routes with common rapid movement along and sudden directional changes.

\section{Random Waypoint Model}

According to the RWP mobility model [3], [52], [42], a MN moves at constant speed and on a straight line between two Waypoints, WP (i.e., random locations in the selected area of movement). By reaching a WP, the node will choose a new $\mathrm{WP}$ and a new speed, according to given distributions. The MN can also wait a certain time before moving on to next WP; however, if pause time is set to zero we actually obtain a movement very similar to random walk). The movement of a MN from a starting WP to its destination WP is called in the literature movement epoch, transition, or movement period.

The RWP and Random Walk models provide non-uniform spatial distribution of nodes [7]. Other related work [52], [6] list some statistical properties and possible flaw results when those two simplest models are used in simulations. 
In particular, there is much higher probability of the node to be in the middle of the modelled area than near to its borders. Some modifications to the original RWP have been proposed, e.g. the Random Direction Model [3], [13], that overcomes the non-uniform spatial distribution problem and provides more distributed paths. Another problem of the Random Walk and RWP is the emulation of sharp turns or rapid speed changes that do not happen for real objects, which have some inertia. However, as further detailed in Section 3.1, the RWP is one of the most used mobility models in simulation studies [1], [49].

\section{Gauss-Markov Mobility Model}

The Gauss-Markov Mobility Model takes into consideration how speed changes in time and smooths out rapid changes of speed and direction during subsequent iterations. Contrary to the RW and RWP, the Gauss Markov model is not memoryless. It assumes that a MN has an initial speed and direction, and takes this fact into consideration while computing these values for the next step:

$$
\begin{gathered}
S_{n}=\alpha S_{n-1}+(1-\alpha) \bar{S}+\sqrt{\left(1-\alpha^{2}\right)} S_{x n-1} \\
d_{n}=\alpha d_{n-1}+(1-\alpha) \bar{d}+\sqrt{\left(1-\alpha^{2}\right)} d_{x n-1}
\end{gathered}
$$

where:

$\alpha$ - tuning parameter

$\bar{S}$ and $\bar{d}$ are mean values of speed and direction

$S_{x n-1}$ and $d_{x n-1}$ are Random Variables from Gaussian distribution

The turning parameter $\alpha$ is used to vary the randomness of changes. There can be 3 cases:

$$
\left\{\begin{array}{l}
\alpha=0 \\
0<\alpha<1 \\
\alpha=1
\end{array}\right.
$$

$\alpha=0$ means that the model has no memory and the movement is totally random, $\alpha=1$ means strong memory which results in linear movement. The ideal case is when $0<\alpha<1$ which means that the model has some memory and the randomness is intermediate - no sharp turns and rapid directional changes. The Gauss Markov does not only model different types of movement depending on its memory strength, but also includes a Brownian motion model when $\alpha=0$.

$S_{x n-1}$ and $d_{x n-1}$ are calculated based on the previous speed and direction. In time moment $\mathrm{n}$ its position is calculated:

$$
\begin{aligned}
& x_{n}=x_{n-1}+S_{n-1} \cos d_{n-1} \\
& y_{n}=y_{n-1}+S_{n-1} \sin d_{n-1}
\end{aligned}
$$

where:

$\left(x_{n}, y_{n}\right)$ - MN's coordinates in time moment $n$

$\left(x_{n-1}, y_{n-1}\right)$ - MN's coordinates in time moment $n$

$\left(S_{n-1}, d_{n-1}\right)$ - MN's speed and directions in time moment $n-1$ 
To make sure that the MN does not leave the simulation area, or it will not stay close to simulation space borders for too long, the $d$ parameter is forced to change by $180^{\circ}$ when the node is close to simulation space borders. The GaussMarkov model solves the problem of rapid speed and direction changes unlike the RW and RWP models, however it still lacks the ability to mimic actions like grouping or avoiding obstacles.

\section{Truncated Levy Walk Model}

While researching the traces of foraging animal patterns [24][46], the authors revealed clear Levy flight characteristics in their movements (a flight is a Euclidean distance between two waypoints visited in succession by the MN). The research was followed by studies on human movement traces [38][39][18], [17][11] which gave some interesting results. According to these studies, human movement characteristics are quite similar to one of the characteristics found in movement of jackals [2] and spider monkeys [37]. To simplify, the Levy walk is a series of short trips with occasional longer ones.

The simple Truncated Levy Walk (TLW) Model [8] that gives heavy-tailed characteristics of human motion. The model is described with 4 variables $\left(l, \theta \Delta t_{f} \Delta t_{p}\right)$ where:

1 is a heavy-tailed variable that describes the flight length. It is obtained from the Levy distribution:

$$
p(l) \sim|l|^{-(1+\alpha)}, l<l_{\max }
$$

$\theta$ is the movement direction taken from $(0,2 \pi)$

$\Delta t_{f}>0$ is the flight time

$\Delta t_{p}$ is the pause time that follows a truncated power law

$$
p\left(\Delta t_{p}\right) \sim\left|\Delta t_{p}\right|^{-(1+\beta)}, 0<\Delta t_{p}<l_{\max }
$$

Using this simple model, the authors were able to obtain synthetic traces with the power-law distribution of inter-contact times. The authors related their research to rather small sets of traces on a bounded space. The traces which they generated using the TLW are quite similar to the collected real traces [39].

\section{Group Mobility Models}

There are several models used for modelling group mobility. The Column Mobility Model represents a movement of several MNs in a line towards a common goal in an orderly fashion. The authors of [3] suggest that such movement can be used as searching and scanning activities of military robots in, for example, mine searching. If the $\mathrm{MN}$ is due to leave the simulation area, similarly to the Gauss Markov model, its direction is flipped by $180^{\circ}$. The next example of group mobility is the Pursue Model, where one node moves freely according to, for example, the RWP and the other nodes are "pursuers" which follow the "escaping" node. This model can be used in scenarios for race, tracking or police pursuit.

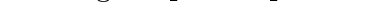


The nomadic Community Mobility Model introduces a group movement, where a group of MNs move together towards a common waypoint, however individual nodes can deviate from the original, straight-to-goal path and move using, for example, the RWP in the vicinity of the moving group. Such behaviour can be observed during organized tours in a zoo or a museum, where the whole group follows a general sightseeing pattern, however individual people move independently inside the group and move toward different points of interest.

\subsection{Social Mobility Models}

Barabási et al. analysed human movement [4], showing that there is an increasing evidence that the human activity routine exhibits properties that follow the nonPoisson model, as the traces could be characterized by bursts of rapidly occurring events separated by long periods of inactivity. Hence, this was the first proof that humans do not move randomly.

Another trace analysis, which was based on a data set obtained from over one million users for several years [11], showed that such traces follows the powerlaw characteristics. Although the traces were exhaustive, they represent mobile roaming users in specific settings and as such we cannot apply such analysis to all potential movement cases. A third study comprises monitoring of movement of 100,000 people, also based on cellphones [18]. Such a study has proven that travel patterns of an individual human follow simple reproducible patterns in spite of the diversity of roaming.

Social mobility modelling considers movement aspects derived from the way humans move and hence, derived from social behavior. For instance, moving to a restaurant implies an interest. The relevancy of such interest impact speed (e.g. acceleration); social interaction impacts the direction as well as stationary time of a node in specific places.

In social mobility modeling, a relevant aspect is the daily routine of citizens, as it has been observed, as described, to have some repeating features. The second relevant aspect is that while moving, users are often involved with other users, be it passively or actively. Humans as social beings are always part of a group which can be virtual in the sense that there may or may not be an association between humans. From the mobility modelling perspective, the relevant aspect to consider is a correlation between the space and time, where different and possibly not acquainted persons cohabit the same space and time due to a temporary (or permanent) routine overlap. For instance, this is the case of two strangers who regularly share the same subway line for a specific period of time every week. To simplify, individual movement exhibits a correlation between time and space. When different people exhibit a similar correlation, then it means that they form a cluster of nodes with the same group (social movement). From the networking perspective, this correlation is relevant to model time and space. 


\section{Sociological Interaction Mobility for Population Simulation - SIMPS}

SIMPS [10] is a mobility model that incorporates recent findings in sociological research on human interaction, assuming that each person has specific social needs derived from their societal posture, as well as assuming that people interact to meet their specific social needs. These two features lay the groundwork for the SIMPS behavioural model which is based on two social actions: socialize - to move toward acquaintances - and isolate - to escape from the undesired presence.

The MN contacts are modelled using a social graph, which represents relationships among people. While the graph does not give information about human proximity, people that are socially attracted tend to meet more often. SIMPS does not model grouping behaviour, however the individual mobility patterns tend to naturally converge, and give collective motion features.

\section{Community-based Mobility Models - CMM and HCMM}

The Community-based Mobility Model (CMM) [35] follows the notion of social attractiveness ( $S A$ ), which has again its roots in the relevancy parameter of social networking. This notion was the predecessor of the SIMPS socialize notion, being a more simple translation of a social belief into a networking parameter. Social attractiveness is a product of the nodes that are, at a specific moment in time, in such a cell. The social attractiveness is computed based on the sum of the cost of associations between $i$ and each of the nodes $j$ in the cell, $w_{i, j}$ This sum is then normalized by the number of nodes associated with the specific cell, $n$. If the cell $S_{p, q}$ is empty, then $\mathrm{SA}$ is also set to 0 . It should be noticed that this function in the CMM serves the sole purpose of selecting the targetcell based on the group of nodes residing in it at a given moment in time. In other words, the function does not determine the node speed nor does it directly determine the node movement pattern, which is simply linear. Considering the deterministic mechanism, the cells are simply selected based on their overall social attractiveness. The probabilistic mechanism is more realistic, given that the selection of the next target becomes proportional to the social attractiveness of each cell for a specific node $i$, based on the roulette-wheel selection, giving the possibility to choose an empty cell. While the CMM is interesting, it still falls short in aspects, such as proximity, considering that humans prefer short distances to long ones, pause time and collision avoidance.

Following the CMM structure, nodes' movement works in the following way: for a node $i$ located in a specific cell $S_{p, q}$, the computation of the next target involves computing the Social Attractiveness (SA) towards node $i$ for each set of nodes positioned in different cells. The SA function is provided in Equation 8 .

$$
S A_{p, q_{i}}=\frac{\sum_{j \in C_{S_{p, q}}} m_{i, j}}{\omega}
$$

Where:

$S A_{p, q_{i}}$ - the social attractiveness towards the MN $i$

$C_{S_{p, q}}$ - the set of the hosts associated to square $S_{p, q}$ 
$m_{i, j}$ - the interaction between two individuals, $i$ and $j$

$\omega$ - the cardinality of $C_{S_{p, q}}$ (i.e. the number of hosts associated to the square $\left.S_{p, q}\right)$.

$S A_{p, q_{i}}$ corresponds to the social attractiveness that a specific set of nodes has towards the node $i$, which in a way measures the social relevancy (the attractiveness enacted) of such a cell to the node $i$. Such attractiveness is a product of the nodes that are, at a specific moment in time, in such a cell. $S_{p, q}$ represents a cell in the grid, $p$ being the row and $q$ being the respective column representation. $n$ corresponds to the total number of nodes in $S_{p, q}$, while $w_{i, j}$ represents the cost of the association (social attractiveness) of the node $j$ in the cell $S_{p, q}$ towards the node $i$. This weight is in fact related to the potential time and space correlation of nodes, which is called social interaction.

The Home-cell Community-based Mobility Model (HCMM) [9], is based on the social model of the CMM but it presents a different approach to model movement. In the HCMM, nodes are attracted by their home cell (the specific community's cell). The nodes are attracted to places swarmed with other nodes from their community. Occasionally, they visit other communities and then return home, so the HCMM reproduces an environment where nodes are attracted towards places selected from places which are popular among their friends.

\subsection{Hybrid Models}

Hybrid models combine various features of human movement, such as its limited randomness, social ties and daily routines. They try to merge the random and social models. Since this approach is relatively new, in this section we present four most interesting propositions of the human movement modelling.

\section{Self-similar Least-Action Human Walk - SLAW}

SLAW [32] is a mobility model based on traces, where the authors extracted some important features from the collected data to define the behaviour of nodes. The pause time and flight length should represent the truncated power-law behaviour. The waypoints can be modelled by fractal points, assuming that people are always more attracted to more popular places.

The main difference between this and the previous model is the community behaviour. Despite the fact that the SLAW is a complete mobility model, we cannot classify it as a social mobility model, given that the movement of nodes is not related to a social parameters. The authors consider correctly that the fact that nodes are at the same time in a specific place does not interfere with their behaviour, hence we do not consider the social mobility model, but the realistic mobility model. The topology of the SLAW model is defined by the Delaunay triangulation on the fractal waypoints using the Brownian Motion generation technique $(\mathrm{fBm})$. After the waypoints are defined over a 2-D area, the movement of nodes is defined by the LATP (Least Action Trip Planning) algorithm, where the distance between all unvisited waypoints is calculated (people choose their 
destination based on the distance), defining the order in which these points will be visited, given that the nearest points should be visited first. The authors assign a weight to each cluster, proportional to the total number of waypoints. Each user chooses from 3 to 5 clusters randomly based on a given probability, proportional to these weights. The main objective in the allocation of weights is the ability to build some sense of community, making the choice of the same clusters possible for many users. To increase randomness, each day a node picks additional waypoints from a different cluster, also respecting from $5 \%$ to $10 \%$ of waypoints in the new cluster. While nodes are in motion, the model does not have significant features, given that acceleration or any other feature that allows nodes change their movement are considered. To finalize the SLAW structure, when a node reaches each waypoint (its destination), it pauses for a period of time which is predefined, considering that the whole trip should be completed in 12 hours ( $\mathrm{T}=12$ hours) and that the node comes back to its starting point within this period.

While SLAW points out that inter-contact times can be modelled by a truncated power-law distribution with the power-law head and the exponentially decaying tail after a certain time, other work proposes the cut-off period to consist of roughly 12 hours. The Working Day Movement the authors simulate a real-life situation of the "going to work-staying at work-going home" cycle. we can see that the exponential cut-off appears roughly after 12 hours, what could suggest the influence of people daily routines on the inter-contact times. This model can be characterized by three activities: working, staying at home, evening activities with friends. These three main activities are connected via the transport submodel. The daily routine is as follows: each node starts its "life" in a fixed location marked as home location. Home is a place which obviously simulates home activities, e.g. the mobile device stays in one place until the wakeup time. After wakeup, the node travels to work where it spends a certain amount of time (which can be configured adequately). During the office hours, the node walks short distances along the office waypoint to simulate movement around the office (this is simulated by an office activity submodel). After the office hours, the node can choose to participate in evening activities or return home. In case of going home, the node stops at a home location, moves a small distance to simulate entering the home and stays inside until wakeup. Evening activities are considered group activities done after work. The node can choose to move to its favourite meeting point, wait there for all other nodes from a group to join in. The group moves together randomly. After these activities the group splits and the nodes return to their home locations. Travelling between activities is done by a transport submodule, which includes a car, bus or a walking submodule. The node that does not own a car chooses whether it travels by a bus or walks. The WDM can be further improved by additional real-life features such as pause time, clear definition of a workday/weekend routines, speed variations in all the travel submodels. Also, if the submodels of vehicular movement are part of the model, it is crucial to include a set of rules of vehicular movement different than walking (the only difference in the model is the speed of travelling). 


\section{The Hybrid Mobility Model based on Social, Cultural and Language Diversity}

This hybrid model [29] is based on a framework which integrates social, cultural and linguistic factors which impact human mobility, including the temporal and spatial features in the model. The model is based on real traces obtained from the IEEE INFOCOM 2006 conference [23], [16]. The data was collected using the IEEE 802.15.4 or 802.11 communication protocols and devices. It includes information about the participant's nationality, spoken language and the number of contacts with other participants. Based on these pieces of information, the authors created a contact graph which represents forming of social activities of groups, communities and participants depending on their social and cultural features. The most common features for MNs are grouped into clusters that impact human mobility, e.g. demographic information (country of citizenship and residence, language) and professional information (affiliation, area of expertise). The model uses the individual MN's features and preferences like the spoken language and citizenship, but also takes into consideration the influence of high -impact groups (like the most popular language group) on the mobility pattern. Although the idea of modelling mobility this way is interesting, still it is based on traces obtained from a very characteristic event, like a conference, and there is no data how such a model relates to everyday situations.

\section{Prediction Applied to Cellular Networks}

In cellular networks, a layout for the BSs to which the mobile devices are connected must be designed. There are several well established patterns, including hexagonal (typical for suburban areas), Manhattan (urban) and linear (highways). The possibility of having several connection layers must also be considered, e.g., microcells as the first choice and macrocells as umbrellas for overflowing traffic. The problem of the cell borders is related to the layout. For simplicity, it is possible to consider a network in which each device is covered only by the BS closest to it. This assumption is simple and allows direct conclusions; however, it is not realistic. When the radiation patterns and random nature of the radio channel are taken into account, the borders are not regular shapes and may change along time (i.e. cell breathing, random fading). This increases the complexity of the analysis significantly; however, it offers realistic results, although they are more difficult to interpret.

The mobile behaviour of devices within the cellular network can be characterized in a variety of ways, with each of them corresponding to a different scenario and environment. Experimental research has been conducted during the last two decades to determine the statistical properties of each representative user class, e.g., public networks, Local Area Networks (LANs), indoors, outdoors, cars, pedestrians, etc. Depending on the network technology, geographical area and type of user, it is possible to characterize users' movements in such a way that the resulting pattern will be similar to the real one. Mobility models play a key role in the analysis of planning issues in a cellular network. Knowledge 
about the pattern followed by the MNs in a given scenario may help network planning to guarantee service along the pathway followed by each user.

The purpose of a cellular network is to provide coverage to moving users while their connections are transferred between cells. As the user moves, the quality of the signal received from the current cell may decrease below the acceptable threshold. This event will trigger the HO procedure [43]. The time needed to transfer the ongoing communications to the new cell is crucial since if this process lasts too long, the user may suffer degradation in the quality of the ongoing services. In some cases, it may lead to a drop in the communication [26]. This issue is particularly important for delay-sensitive applications such as video and audio streaming. Mobility prediction techniques allow the network to be prepared for the HO in advance (e.g., booking resources for the expected HO in the destination cell) [47]. Resource demands could fluctuate abruptly due to the movement of high data rate users. If one can predict requests of bandwidth at a cell, the overall network performance would improve.

Some research has been targeted to develop models that predict the users' trajectory, which can be used to allocate resources at the new cell and to aid the HO process. The technique proposed in [47] uses real-time mobile positioning information and previous HO locations reported by other nodes in order to predict the $\mathrm{HO}$ event. With mobility prediction, the resources reservation at each BS can dynamically be adjusted. They demonstrate that reservation efficiency improves as the knowledge incorporated into the scheme increases. Joshi et al. [27] aim to exploit the notion of predictability and propose algorithms to achieve low latencies while minimizing the message overhead. Prediction of users' movement is also possible using trace data [33]. For HO success, the most important predictions occur when moving into highly populated cells. Other studies exploit real traces to analyse the predictability of users' movements inside the network. Sricharan and Vaidehi [48] examine real-time mobility traces and identify key mobility parameters. A generic framework for mobility prediction is described in [36], where a model is proposed to predict the sequences of user's paths from observed sequences.

Structural approaches study mobility through mathematical models that try to represent human or vehicle mobility. Mobility models play a key role when planning a wireless network (i.e., resource allocation, location updating, and channel holding time). The RWP mobility model is simple and has extensively been used in simulation studies. Kurkowski et al. [31] analyse MANET simulation studies published in a premiere conference for the MANET community between 2000 and 2005 . They found that $66 \%$ of the studies involving mobility used the RWP mobility model. Despite it has been criticized for not being representative of how humans actually move, it is still largely used in many studies [1], [49]. Rojas et al. [40] validate the RWP against real mobility data. With small changes to the distributions used in the model (e.g., non-uniform distribution of the waypoints), the authors show that it can be used as a good model for mobility in large geographic areas such as a city. Considering that extracting a mobility pattern from real traces is complex and, anyway, it would be specific to the 
environment and conditions from which it has been extracted, the use of the RWP mobility model in simulation studies is widely accepted.

Recently, a detailed description of the analytical framework to forecast the next cell to which a user moving according to the RWP mobility model handovers within a certain period of time has been provided [54]. The details of the movement pattern according to the RWP are given in Section 2.1. The movement between two WP is called a leg and its length is represented here by $l$. The assumption behind the proposed framework is that the user knows its current position and the position and time of the last change of speed and direction (i.e., the last WP). This analysis follows previous research on statistical characteristics of the RWP mobility model (i.e., length and duration of the straight movement between two waypoints [6], node distribution [25]). By assuming a symmetrical layout (i.e., any symmetrical layout is valid, e.g. 4 APs providing coverage to a circular area as presented here), the framework is able to predict the HO to each APs in a given interval of time. This time interval $\Delta t$ is short enough so to guarantee that the node will reach no more than one waypoint $(X(\Delta t) \leq 1)$. In this way, the probability of $\mathrm{HO}$ in $\Delta t$ can be written as the sum of the probability of HO in case a WP is reached during $\Delta t$ (which we call $\operatorname{Pr}\{H O a\})$ plus the probability of HO in case the node does not change direction and speed during $\Delta t$ (which we call $\operatorname{Pr}\{H O b\}$ ), as shown in equation 9:

$$
\begin{aligned}
\operatorname{Pr}\{\mathrm{HO} \text { in } \Delta t\}= & \operatorname{Pr}\{X(\Delta t)=1\} \cdot \operatorname{Pr}\{\mathrm{HO} \text { in } \Delta t \mid X(\Delta t)=1\}+ \\
& \operatorname{Pr}\{X(\Delta t)=0\} \cdot \operatorname{Pr}\{\mathrm{HO} \text { in } \Delta t \mid X(\Delta t)=0\}= \\
& \operatorname{Pr}\{X(\Delta t)=1\} \cdot \operatorname{Pr}\{H O a\}+\operatorname{Pr}\{X(\Delta t)=0\} \cdot \operatorname{Pr}\{H O b\}
\end{aligned}
$$

The following parameters are considered:

$-\gamma_{j}$ : represents the current angle of direction and $v_{j}$ the current speed.

- $Y$ is the distance between the last WP and the current position $\left(P_{t}\right)$ at time

$t$ (i.e., the distance already travelled at speed $v_{j}$ in direction $\gamma_{j}$ ).

- $x_{t}$ is the distance of $P_{t}$ from cell boundaries in current direction $\gamma_{j}$.

$-x_{A}$ is the distance of $P_{t}$ from whole area in direction $\gamma_{j}$.

The flow chart in Fig. 1 provides the algorithm used to estimate the probability of $\mathrm{HO}$, the probability of no $\mathrm{HO}$ and the expressions used in each case. It is easy to understand that $\operatorname{Pr}\{H O b\}$ is easy to evaluate if the geometry of the layout and the current position, speed and direction of movement are known (as assumed). A more complex calculation is needed for $\operatorname{Pr}\{H O a\}$, instead. The correspondent equations and a brief explanation are given hereafter. Different formulations can be applied to $\operatorname{Pr}\{X(\Delta t)=1\}$ or $\operatorname{Pr}\{X(\Delta t)=0\}$ according to the geometry (i.e., relationship among $x_{t}, x_{A}$, and $\Delta t \cdot v_{j}$ ), as shown in Fig. 1.

The final expression for the probability of HO given that one WP is reached during $\Delta t$ is given by Equation 10 .

In equation $10 \operatorname{Pr}\{H O a \mid r\}$ represents the probability of $\mathrm{HO}$ given that the next WP is at the generic point $S(r)$ at distance $r$ from $P_{t}$, which is reached 


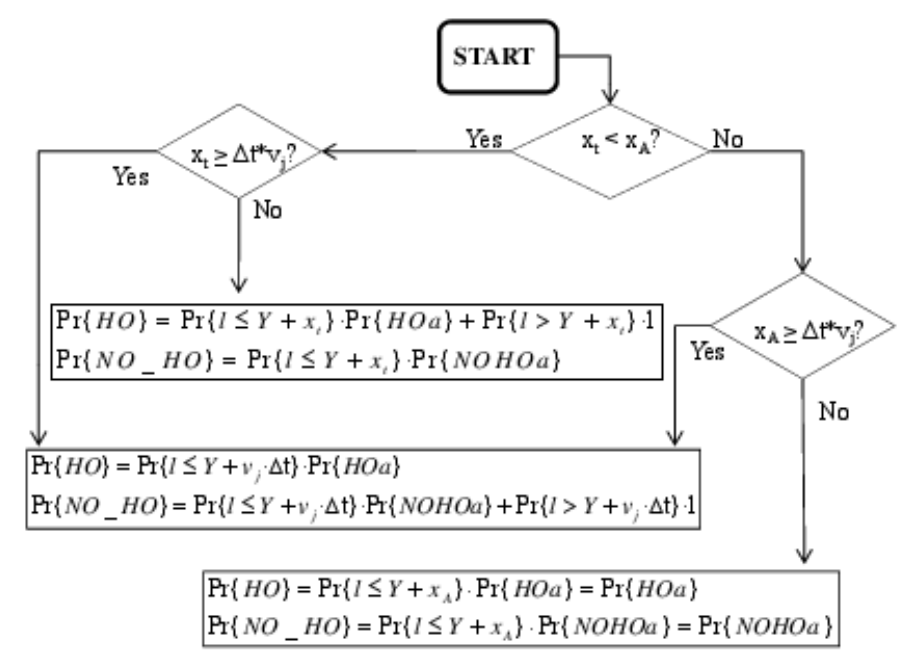

Fig. 1: Formulas used to determine the probability of HO and NO HO.

at the generic time $\left(t+r / v_{j}\right) ; f_{R}(r)$ is the pdf of the possible positions $S(r)$. A graphical example of how to evaluate $\operatorname{Pr}\{H O a \mid r\}$ in case $S(r)=P_{t}$ (i.e., $r=0$ ) is provided in Fig. 2. Two circles can be drawn around $S(r)$, each representing the distance reached in any direction if the minimum speed (red circle) or maximum speed (blue circle) is selected. Then, the points falling inside the crown and the area of movement (black circle) represent all the possible locations that can be reached in $\Delta t$; those falling outside the coverage of AP1 (black discontinuous line) represent the probability of HO (green area).

Simulations have been run to validate the proposed analytical model and to test the loss of accuracy produced by the necessary simplifications to the analytical model. Numerical results and simulation results are presented for ten different cases in Table 1; values in italic correspond to the simulation results. For each scenario, the probability of HO to AP1, AP2, AP3 and AP4 are displayed $(\operatorname{Pr}\{\mathrm{HO} 1\}, \operatorname{Pr}\{\mathrm{HO} 2\}, \operatorname{Pr}\{\mathrm{HO} 3\}$ and $\operatorname{Pr}\{\mathrm{HO} 4\}$, respectively). Values in square brackets stand for the probability of remaining in the current cell (i.e., $\operatorname{Pr}\{$ NO$\mathrm{HO}\}$ ). It is shown that the error is always smaller than $2 \%$. The importance of this study is twofold. First, it provides a deeper insight into the statistics of the RWP mobility model, extending previous analysis. This statistical knowledge provides a better understanding of the interplay of the mobility pattern with network parameters. Second, and from a more practical perspective, the HO prediction is useful to manage resource allocation and reservation strategies. For example, these results can be applied in studies on allocation strategies for QoS improvement in cellular networks. 


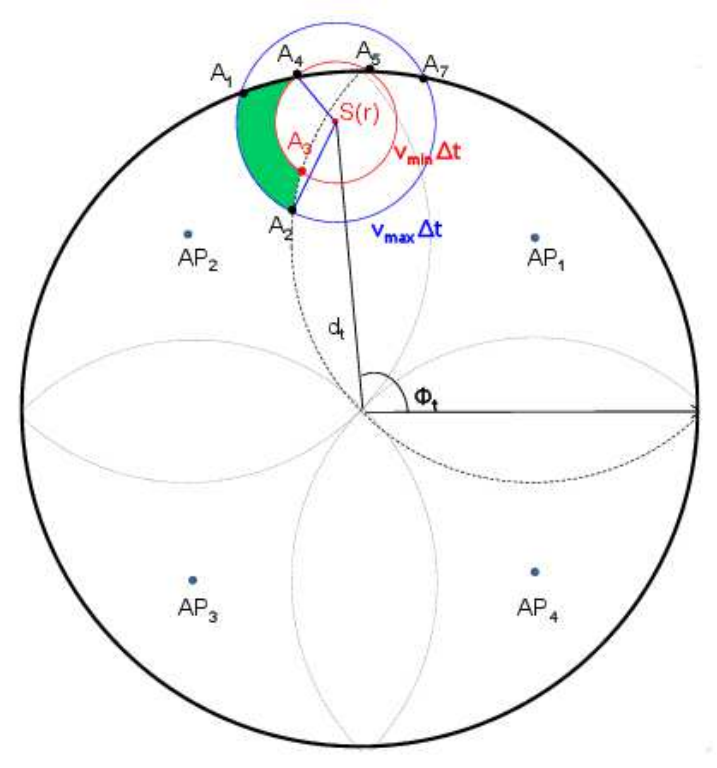

Fig. 2: Area representing the $\operatorname{Pr}\{H O a \mid r\}$ when $S(r)=P_{t}$. At time $t$, the node is associated with AP1.

The model has been also applied in a simulation study through which the implications of different HO strategies (i.e., aggressive vs. conservative) and of more complex radio patterns (i.e., shadowing) can be analysed [53]. Under non-ideal channel conditions, the HO probability generally increases when the shadowing effect is taken into account; the shadowing has an effect on the borders of the current cell which now change over time, thus making higher probable that the node exits its cell if compared to the ideal channel situation. However, depending on the geometry, shadowing can result in a "capture effect" in the current cell, especially when the node is located close to the cell borders. Moreover, it has been proven that the impact of the HO strategy is stronger in non-ideal channel conditions [53].

\section{Alternative Methods for Simulation of Changes in Radio Signal Propagation}

The transmission performance in mobile wireless networks is determined by two dominant factors: the bitrate of the communication and the packet loss ratio. Typically, locations of access points and BSs are given. The locations of clients are emulated by the mobility model. The radio signal propagation model, such as Okumura-Hata, together with the MAC layer simulation are used to calculate the effective data throughput and packet loss rate. We try to simplify this by creating a model that generates the bitrate and the packet loss ratio directly. 
Table 1: Numerical and simulation (italic) results for the ten cases analysed. Values in square brackets stand for the probability of remaining in current cell.

\begin{tabular}{|c|c|c|c|c|c|c|c|c|}
\hline & \multicolumn{2}{|c|}{$\operatorname{Pr}\{$ HO1 $\}$} & \multicolumn{2}{|c|}{$\operatorname{Pr}\{\mathrm{HO} 2\}$} & \multicolumn{2}{|c|}{$\operatorname{Pr}\{\mathrm{HO}\}$} & \multicolumn{2}{|c|}{$\operatorname{Pr}\{\mathrm{HO} 4\}$} \\
\hline 1 & 0.83 & 0.64 & {$[98.28]$} & {$[98.76]$} & 0.83 & 0.58 & 0.06 & 0.02 \\
\hline 2 & 0.62 & 0.46 & 7.43 & 98.08 & 0.62 & 0.49 & 1.32 & 0.98 \\
\hline 3 & & {$[73.80$} & & 0.00 & 0 & .00 & 9.94 & 26.2 \\
\hline 4 & .90 & 99.65 & & 0.08 & 0.04 & 0.11 & {$[0.04]$} & $\overline{[0.16}$ \\
\hline 5 & [100] & {$[100]$} & 0.00 & 0.00 & 0.00 & 0.00 & 0.00 & 0.00 \\
\hline 6 & 0.75 & 0.35 & {$[98.46]$} & {$[99.21$} & 0.78 & 0.44 & 0.01 & 0.00 \\
\hline 7 & {$[20.77]$} & [22.02] & 68.20 & 70.76 & 2.04 & 0.96 & 8.99 & 6.27 \\
\hline 8 & {$[3.78]$} & [2.73] & 90.43 & 93.66 & 2.48 & 1.20 & 3.31 & 2.40 \\
\hline 9 & 0.02 & 0.01 & 0.14 & 0.06 & $8.66]$ & {$[15.06$} & 81.18 & 84.8 \\
\hline 0 & [63.85] & {$[57.00]$} & 19.59 & 25.30 & 5.35 & 3.21 & 11.21 & 14.50 \\
\hline
\end{tabular}

We based our work on the GPS traces gathered from multiple travels along the same path by the same person. This provides a good representation of the rate of changes during a typical way to work, or a travel on a predefined path. We defined some location of the Wi-Fi access points and calculated the distributions of time in which the client enters the range of the access point and crosses the borders of areas with different signal levels. To model an effective data rate, the AP coverage is divided into areas of relatively constant transmission conditions representing an area in which a specific modulation (and effectively bitrate) is used. The measured distributions of the passage time per area were matched with common statistical distributions, and showed to fit into normal distribution in most cases. In some cases, the location of the AP influences heavily the distribution of change time, e.g. when an AP is located near the traffic lights, the change must be modelled using the bimodal or multimodal distribution. The model can be parameterized to represent such locations correctly. The model is verified by comparison to the GPS, signal level and transmission rate traces gathered in the real life conditions.

\subsection{Proposed Model of Wireless Network Throughput}

We assume that within the area on which a single coding rate is used, a client is offered a constant throughput which depends only on the packet size and the number of other clients connected to the same AP. This is a simplification based on assumptions made already by most of the discrete event simulation models, such as neglecting the influence of interferences. To determine the data rate offered to a client, we need to define the moments at which it enters and leaves the area with a particular coding rate. We used the distances provided in [41].

In the simulation model, we propose the transmission time of a packet is determined by the client bitrate (which is selected according to the area in which currently the client is). A guard time representing the MAC layer activity is added between two consecutive packet transmissions. It is calculated based on 
the packet size and the number of clients connected to the same AP, according to the values provided in [15]. When multiple clients are using the same AP, the traffic is served using the round robin scheme, which represents a fair allocation of radio resources.

To select moments at which clients move into or out of the area with a specific coding rate, we gathered GPS traces of multiple travels along a single path by the same person. The GPS traces are used to collect the distribution of passage time between points in space, which represent the change in transmission conditions (the moments of entering and leaving the area). The points in time have been selected according to the signal propagation model to calculate the maximum and minimum distance at which a specific coding scheme is optimal. We defined some location of the Wi-Fi access points and calculated the distributions of time in which the client enters the range of the access point and crosses the borders of areas with different signal levels.

\subsection{Sample Results}

The measured distributions of the passage time per area were matched with the common statistical distributions. In most cases they fit into the normal distribution. In some cases, the location of the AP influences heavily the distribution of the change time, e.g. when the AP is located near to the traffic lights, the change must be modelled using the bimodal or multimodal distribution. Such distributions for a straight road within the AP access are present if there is a road crossing. They are shown in Fig. 3. The model can be parameterized to represent elements like road crossing and traffic lights correctly.

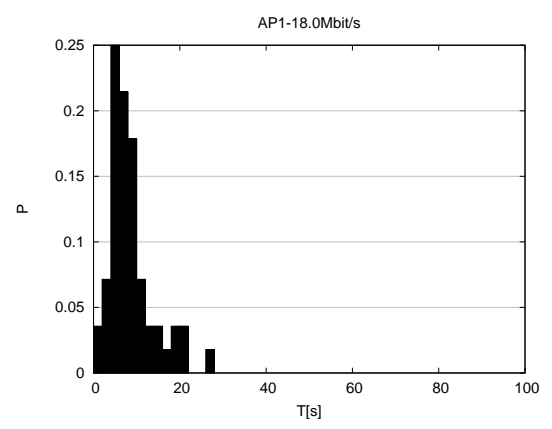

(a) AP1 .

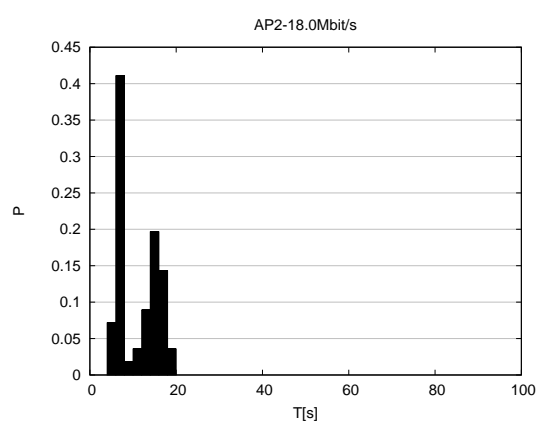

(b) AP2.

Fig. 3: Distribution of the passage time of a single coding rate for a client moving along a straight road in the AP range (a) and for a client moving through a crossing in the AP range (b). 
The GPS trace represents a typical path taken by a single mobile client. It provides a good representation of the rate changes during a typical way to work, or a travel on a predefined path. The sample track that we used in the simulation, with marked points at which the coding rate changes, is shown in Fig 4. It represents a single path for a single client. To model multiple clients we need to use multiple instances of the same node (it will represent multiple clients using the same path) or gather GPS traces from multiple clients.

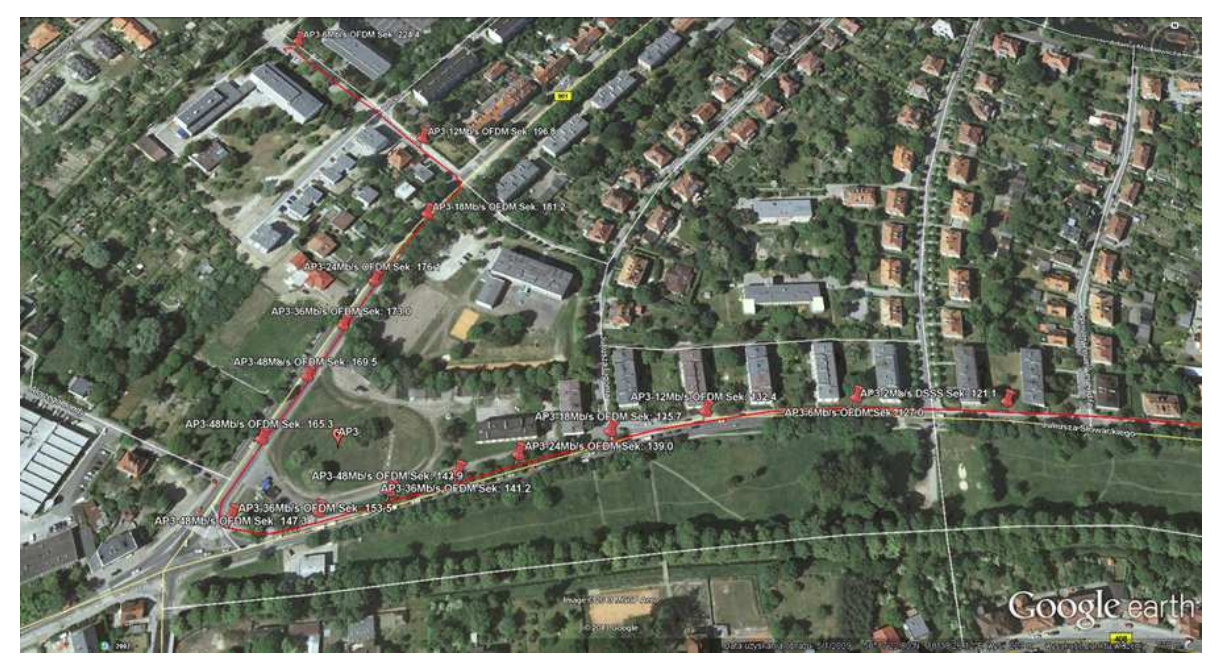

Fig. 4: Map of a simulated AP with the marked path and points at which the coding rate changes.

The results for the model showing the coding rate simulated in time for a sample path is presented in Fig. 5. This is an input for the packet delay model, which also takes into account the packet size and the number of clients connected to the same AP and calculates the packet delay.

\subsection{Validation of the Simplified Model}

To validate the proposed simplified representation of bitrate change, we have created a discrete event simulation model using the ns- 3 environment. The simulation consists of $3 \mathrm{Wi}-\mathrm{Fi}$ Access Points and a single client, which was moving according to the GPS trace imported into the simulation. The Access Points were connected to a PC by a simulated Ethernet link (using a CSMA NetDevice), which was flooding the client with packets. The FreeSpace signal propagation was used.

We have collected the Wi-Fi bitrate of packets transmitted in the simulation and compared it to the bitrate generated by the proposed simplified model.Fig. 6 


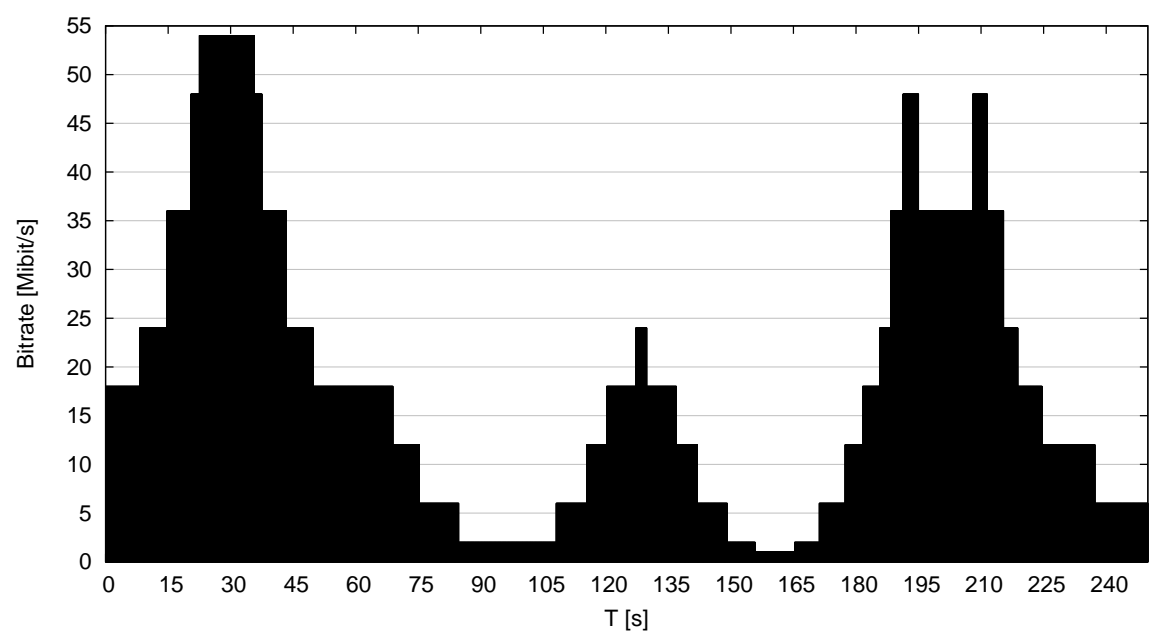

Fig. 5: Sample simulation results with the model of the coding rate changes in time .

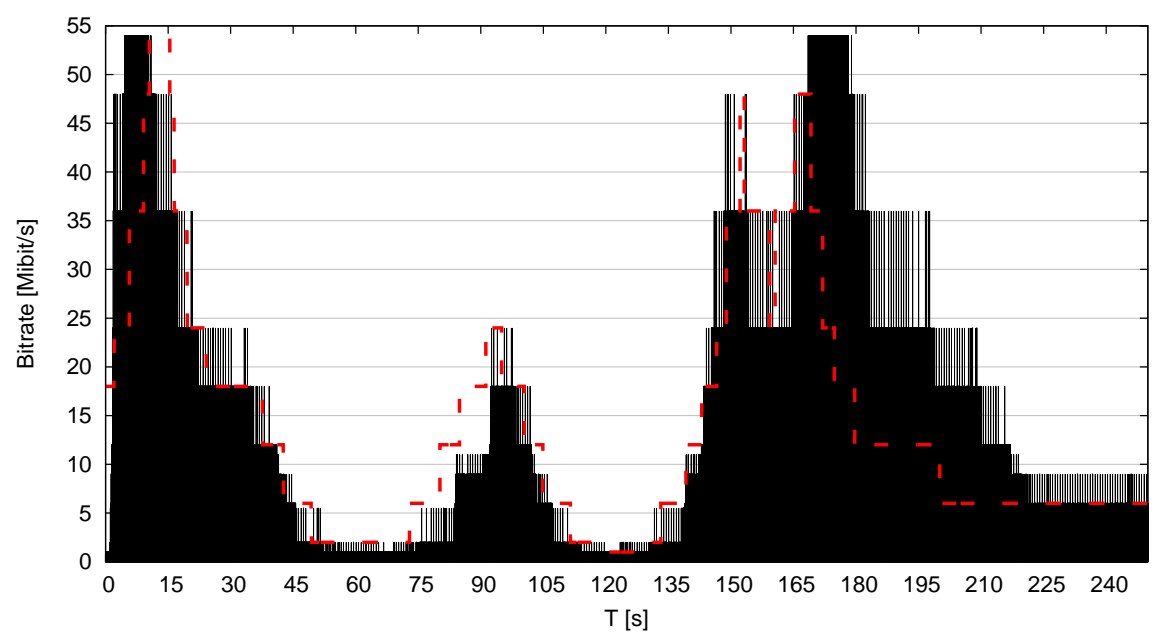

Fig. 6: Comparison of the bitrate generated by the proposed simplified model (red) and the bitrate used in the ns-3 simulation (black).

shows how both bitrates changed in time for sample simulation run. In the simplified models, the results provide similar values of the bitrate in time, however the simulation shows that in some cases the modulation and coding scheme in Wi-Fi jumps constantly between two values, what is not captured by our model. 

promotion or for creating new collective works for resale or for redistribution to thirds must be obtained from the camera-ready copyright owner. The camera-ready version of this work has been published by Springer international, Book Wireless Networking for Moving Objects, 2014,

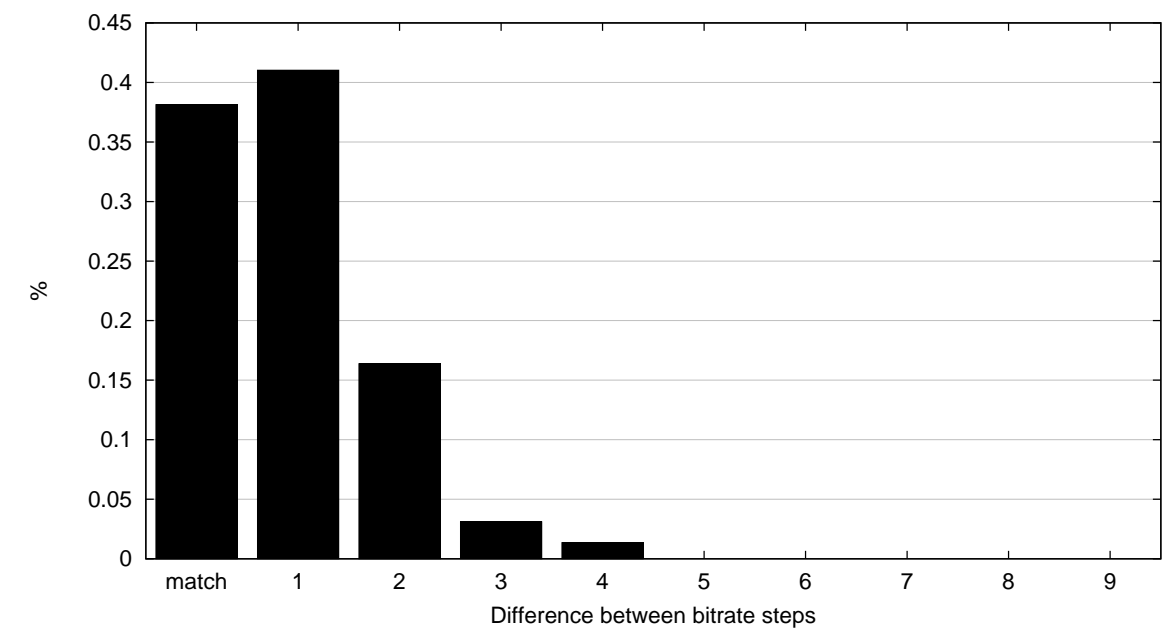

Fig. 7: Distribution of difference in bitrate steps between the simplified and full signal propagation model.

evaluate quantitatively the difference between proposed simplified model and the full simulation with radio signal propagation model we have executed the simulation 30 times for different random seeds over a sample path presented on Fig. 4. We have measured at what percentage of time the biterates provides by both models match, when they differ by one or more steps. The results presented on Fig. 7 show that at $80 \%$ of time the simplified model provided values which were the same of differed only by one step.

\subsection{Computational Complexity}

To evaluate the computational complexity of the model we have implemented three models representing a node moving along the same path: full simulation with packet transmission and radio signal propagation calculations, simulation with packet transmission through channel with modeled bitrate changes and calculation only of the bitrate changes in time. The simulations were executed 30 times for different random seeds. The average execution time of the generation of bitrate changes was below $2 \mathrm{~s}$ for the sample path presented on the map, what is shown on the right bar on Fig. 8. The execution of the simulation with packet transmission over wireless network with emulated bitrate according to the presented model required on average 16 seconds to execute, while the simulation of the full WiFi MAC model with signal propagation calculation required more than 45 seconds (left bar on Fig 8). It shows that the simulation that uses our model is approximately 3 times faster than the traditional method, and the computational complexity of the bitrate calculation is more than one order of complexity lower than the calculation of the full client mobility model: it requires less than 2 seconds comparing to 29 seconds required for the calculation of bitrate 


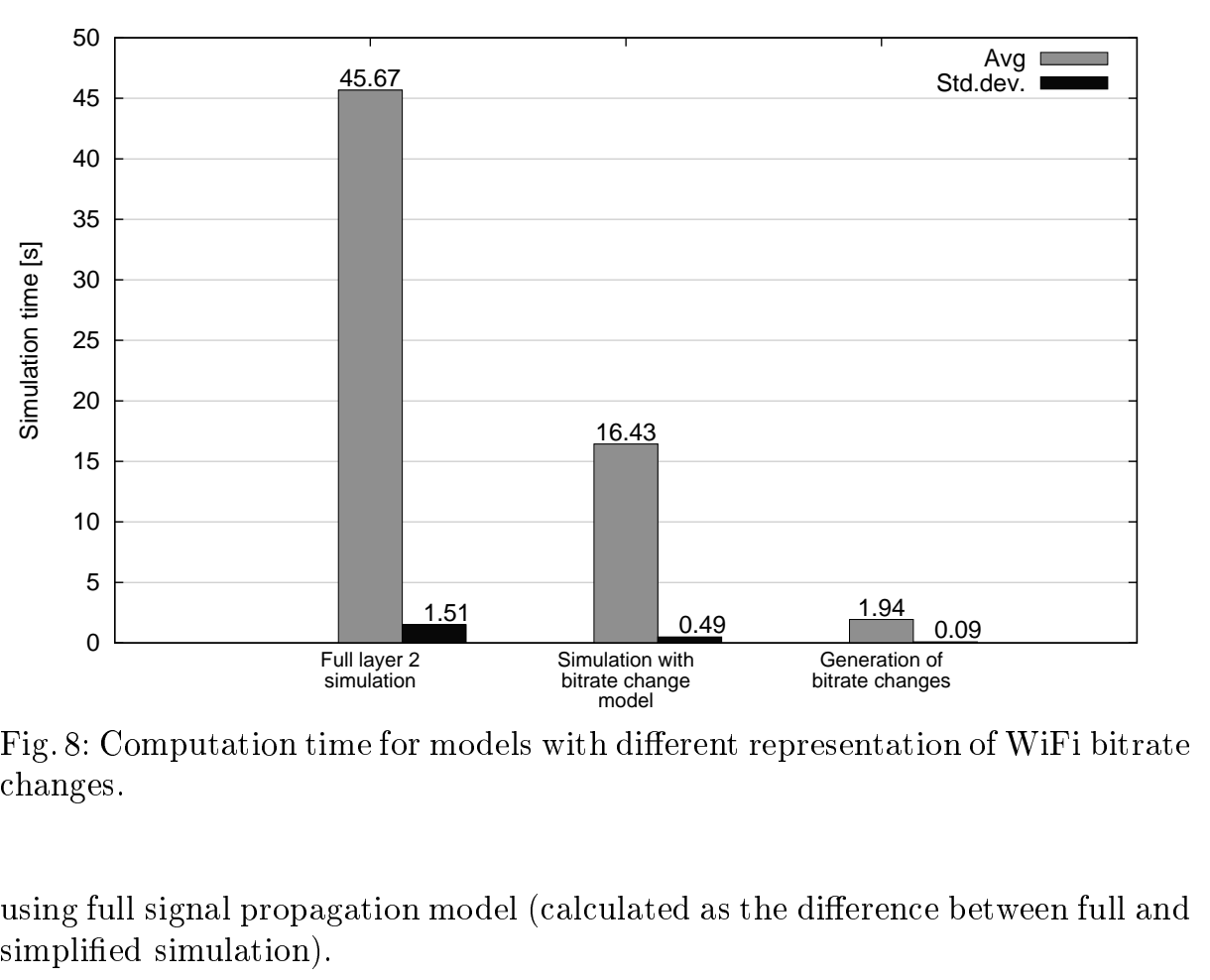

Fig. 8: Computation time for models with different representation of WiFi bitrate changes.

using full signal propagation model (calculated as the difference between full and simplified simulation).

\section{Conclusions}

The problem of mobility modelling is one of the most important parts of the network performance evaluation. Good quality traces are the basis of a network simulation, thus choosing the adequate mobility model for generating these traces is crucial. In this chapter, we briefly presented large variety of mobility models available in the literature. Mobility modelling of mobile devices movement correlates with modelling of a human-like movement and it is still under research. The Hybrid models proposed in section 3.3 are a good basis for modelling such a movement in a cellular network environment.

We also discuss two novel approaches to the mobility models: using it to handoffs optimization and replacing it by a bitrate modelling. In mobile communications, the probabilistic knowledge of the terminals movement and the subsequent prediction of their position are useful to improve several processes such as HO and paging and the signaling associated with them. In this chapter, some advances towards improving these processes have been presented along with the mobility models for synthetic trace generation. An analytical framework for the estimation of the probability of a node handoff to its neighbour cells has been presented. The analysis is useful for simple scenarios, such as RWP mobility, a simple symmetrical layout, position and time of the last known change in transition. The model can be generalized to other symmetrical layouts and the

transition. The model can be generalized to other symmetrical layouts and the (1) 西 (1) 西

西


comparison of the analysis and simulation proves the validity of the analytical framework. The terminal ends up at the expected cell in a significant proportion of predictions, hence the needed capacity for $\mathrm{HO}$ can be correctly reserved most of the times. The above-mentioned prediction algorithm needs to be fed with accurate historical data that reflects the movement and use of capacity by the nodes.

The statistical analysis of actual bandwidth traces for moving terminals and the changes of involved available bandwidth can sometimes substitute the actual traces in simulations. The results presented in this chapter show that changes of the available bandwidth versus movement of the terminal generally follow the common probability distributions. The use of such distributions in the simulations leads to more compact simulation models, requiring less computations. In the section 4 of this chapter the simplified model is presented. It simulates bitrate changes without the need to perform complex computations (running mobility and signal propagation models). The use of such a model can decrease the simulation execution at relatively low costs of slight precision loss.

\section{Acknowledgement}

This work was partially supported by the grant of the Polish National Centre for Research and Development, No. LIDER/10/194/L-3/11.

We thank the team of the User-centric Mobility Management (UMM, http://copelabs.ulusofona.pt/ um project funded by Fundação para a Ciência e Tecnologia, reference PTDC/EEATEL $/ 105709 / 2008$.

\section{References}

1. Ali, T., Saquib, M., Sengupta, C.: Vertical handover analysis for voice over WLAN/cellular network, in Proc. IEEE International Conference on Communications, pp. 1-5 (2010)

2. Atkinson, R.P.D., Rhodes, C.J., Macdonald, D.W, Anderson, R.M.: Scale-free dynamics in the movement patterns of jackals, OIKOS, vol. 98, no. 1, pp. 134-140, $(2002)$

3. Bai, F., Helmy, A.: A survey of mobility models, Wireless Adhoc Networks. University of Southern California, USA 206 (2004)

4. Barabási, A. L., "The origin of bursts and heavy tails in human dynamics", Nature, 207-211 (2005)

5. Bettstetter, C.: Mobility modelling in wireless networks: categorization, smooth movement, and border effects." ACM SIGMOBILE Mobile Computing and Communications Review 5.3, 55-66 (2001)

6. Bettstetter, C., Hartenstein, H., Perez-Costa, X.: Stochastic Properties of the Random Waypoint Mobility Model, ACM/Kluwer Wireless Networks (Special Issue on Modelling and Analysis of Mobile Networks), 10(5), 555-567 (2004)

7. Bettstetter, C., Wagner, C.: The Spatial Node Distribution of the Random Waypoint Mobility Model. Proceedings of WMAN, Ulm pp. 41-58 (2002) 
8. Birand, B., Zafer, M., Zussman, G., Lee K-W.,:Dynamic Graph Properties of Mobile Networks under levy walk mobility IEEE 8th International Conference on Mobile Adhoc and Sensor Systems, MASS 2011, Valencia, Spain, October 17-22, 2011 pp. 292-301 (2011)

9. Boldrini, C., Passarella, A.: HCMM: Modelling spatial and temporal properties of human mobility driven by users' social relationships. Elsevier Computer Communication (2010)

10. Borrel, V., Legendre, F., de Amorim, M.D.: Simps: Using sociology for personal mobility. IEEE/ACM Trans. Netw. 17, 831-842 (2009)

11. Brockmann D., Hufnagel L., Geisel T.: The scaling laws of human travel Nature 439, 462-465 (2006)

12. Cai, L. Chen, R. Sofia.: A Dynamic and User-Centric Network Selection in Heterogeneous Wireless Networks. IEEE International Performance, Computing, and Communications Conference, 2007. IPCCC (2007)

13. Camp, T., Boleng, J., Davies, V.: A Survey of Mobility Models for Ad Hoc Network Research ACM Computing Surveys (CSUR) Volume 37 Issue 2, June 2005, Pages 164-194 ACM New York, NY, USA (2005)

14. Chaoming, S., et al. Science 327, 1018: Limits of Predictability in Human Mobility (2010)

15. Chatzimisios, P., Vitsas, V., Boucouvalas, A.C.: Throughput and delay analysis of IEEE 802.11 protocol, Networked Appliances, 2002, Liverpool, Proceedings, 2002 IEEE 5th International Workshop on, pages 168-174. IEEE (2002)

16. CRAWDAD - A Community Resource for Archiving Wireless Data at Darthmouth, http://crawdad.org/, accessed on November (2012)

17. González, M. C., Hidalgo, C. A., Barabási, A.-L. Nature 453, 779-782 (2008)

18. Gonzalez, M. C., Hidalgo, C. A., Barabasi, A.-L.: Understanding individual human mobility patterns, Nature, vol. 453, pp. 779-782,Jun. (2008)

19. Gorawski, Marcin, Marks, P., Gorawski, Michal: Collecting data streams from a distributed radio-based measurement system. LNCS Volume: 4947, P: 702-705, 2008 (2008)

20. Gorawski, Michal, Grochla, K.: The Real-Life Mobility Model: RLMM. In proc. Of FGCT 2013, London., 12-14.2013 (2013)

21. Einstein, A.: Investigations on the Theory of the Brownian Movement. 1956, Dover Publications Inc., New York (1956)

22. Ekman, F., Keränen, A., Karvo, J., Ott, J.: Working Day Movement Model In Proc. 1st ACM/SIGMOBILE Workshop on Mobility Models for Networking Research (2008)

23. Hossmann T., Spyropoulos T., Legendre, F., "A complex network analysis of human mobility," Computer Communications Workshops (INFOCOM WKSHPS), 10-15 April 2011 IEEE Conference on, pp.876-881 (2011)

24. Humphries, N.E., Weimerskirch, H., Queiroza, N., Southall, E.J., Sims, D.W.: Foraging success of biological Lévy flights recorded in situ

25. Hyttiä, E., Lassila, P., Virtamo, J.: Spatial node distribution of the random waypoint mobility model with applications, IEEE Transactions on Mobile Computing, 5(6), 680-694 (2006)

26. Iraqi, Y., Baoutaba, R.: Handoff and call dropping probabilities in wireless cellular networks, in Proc. IEEE International Conference on Wireless Networks, Communications and Mobile Computing, vol.1, pp.209-213 (2005)

27. Joshi, T., Mukherjee, A., Agrawal, D.P.: Exploiting mobility patterns to reduce reauthentication overheads in infrastructure WLAN networks, in Proc. IEEE Canadian Conference on Electrical and Computer Engineering, pp.1423-1426 (2006) 
28. Karagiannis, T., Le Boudec J-Y., Vojnovic, M., Power Law and Exponential Decay of Inter Contact Times between Mobile Devices, in Proc. ACM MobiCom Montreal, QC, Canada, Sep. 2007, pp. 183-194 (2007)

29. Karim, L., Mahmoud, Q.H.: A Hybrid Mobility Model based on Social Cultural and Language Diversity,9th IEEE International Conference CollaborateCom (2013)

30. Kim, T.S., Kwon, J.K., Sung, D.K.: Mobility Modelling and Traffic Analysis in Three-Dimensional High-Rise-Building Environments, IEEE Trans. on Vehicular Technology, vol.49, No.5, pp.1633-1640 (2000)

31. Kurkowski, S., Camp, T., Colagrosso, M.: MANET simulation studies: the incredibles, SIGMOBILE Mobile Computing Communications Review, 9(4), 50-61 (2005)

32. Lee, K., Kim S.J., Rhee I., Chong, S., SLAW: Self-Similar Least-Action Human Walk. IEEE/ACM Trans. Netw. 20(2): 515-529 (2012)

33. Michaelis, S., Wietfeld, C.: Comparison of user mobility pattern prediction algorithms to increase handover trigger accuracy, in Proc. IEEE Vehicular Technology Conference, vol.2, pp. 952-956 (2006)

34. Musolesi, M., Hailes, S., Mascolo, C.: An ad hoc mobility model founded on social network theory. MSWiM 2004:20-24 (2004)

35. Musolesi, M. and Mascolo, C.:A community based mobility model for ad hoc network research, ACM Press, 31-38 (2006)

36. Prasad, P.S., Agrawal, P.: Movement prediction in wireless networks using mobility traces, in Proc. 7th IEEE Conference on Consumer Communications and Networking Conference, pp. 1-5 (2010)

37. Ramos-Fernandez, G., Morales, J.L, Miramontes, O., Cocho, G., Larralde, H., Ayala-Orozco, B.: Levy walk patterns in the foraging movements of spider monkeys (ateles geof-froyi), Behav. Ecol. Sociobiol. 273, 1743-1750 (2004)

38. Rhee, I., Lee, K., Hong, S., Kim, S. J., Chong, S.: Demystifying the Levywalk nature of human walks, CS Dept., NCSU, Raleigh, NC, Tech. Rep., [Online]. Available: http://netsrv.csc.ncsu.edu/export/Demystifying \_Levy $\backslash$ _Walk \_Patterns.pdf (2008)

39. Rhee, I., Shin, M., Hong, S Lee, K., Chong, S.: On the Levy-walk Nature of Human Mobility: Do Humans Walk like Monkeys? Journal IEEE/ACM Transactions on Networking (TON) Volume 19 Issue 3, June 2011 Pages 630-643 (2011)

40. Rojas, A., Branch, P., Armitage, G.: Validation of the random waypoint mobility model through a real world mobility trace, in Proc. IEEE Region 10 TENCON, pp. 1-6 (2005)

41. Romano. P.,: The range vs. rate dilemma of WLANs, http://www.eetimes.com/ document.asp?doc \_id=1271995 (2004)

42. Roy, R.R: Handbook of Mobile Ad Hoc Networks for Mobility Models 1st Edition., LXIV, 1104 p. Springer; (2011)

43. Sgora, A., Vergados, D.: Handoff prioritization and decision schemes in wireless cellular networks: a survey. IEEE Communications Surveys \& Tutorials, 11(4), $57-77$ (2009)

44. Shen, X., Mark, J.W.,:Mobility Profile Prediction Using Fuzzy Inference in Cellular Networks, invited chapter in Computational Intelligence in Telecommunications Networks, pp.449-477, Editors W. Pedrycz and A. V. Vasilakos, CRC Press (2000)

45. Isaacman, S., Becker, R., Cáceres, R., Martonosi, M., Rowland, J., Varshavsky, A., Willinger, W.: Human mobility modeling at metropolitan scales. In Proceedings of the 10th international conference on Mobile systems, applications, and services (MobiSys '12), ACM, New York, USA, 239-252 (2012)

46. Sims, D. W. et al. Nature 451, 1098-1102 (2008) 
